\title{
COMPARISON OF HOGHORN ANALYSIS METHODS
}

\author{
Vladimir SCHEJBAL ${ }^{1,}$, Vaclav VLASAK2 ${ }^{2}$, Dusan CERMAK ${ }^{1}$, Vitezslav KRCMAR ${ }^{1}$
}

\begin{abstract}
The paper compares an aperture method and full-wave numerical simulations of hoghorn, which is used as a simple primary feed for reflector antennas or individually without reflector. The hoghorn consists of a sectoral horn flaring in only one plane and a parabolic cylinder. We briefly explain the calculations of hoghorn dimensions, aperture distributions and radiation patterns. This is suitable for numerical computations. We analyze the completely new simultaneous comparisons of the ample experiments with numerical results of both the aperture method and the numerical simulations. This enables to obtain innovative conclusions. Proposed improvements can diminish slight differences of one polarization between experiments and numerical simulations. We have used the described methods for the design of certain antennas for multilateration systems, such as Tamara and Vera passive radars, for civilian and military air traffic control, which greatly improve the accuracy of tracking aircrafts.
\end{abstract}

Keywords air traffic control, antenna design, hoghorn, antenna radiation patterns, radar antennas, antenna measurements

\section{INTRODUCTION}

The design of any antenna and especially radar antenna should use suitable software. It is frequent radar prerequisite to have a narrow beam in one plane and a shaped beam in the other. Various primary feeds can produce the fan beams. A sectoral horn with aperture length greater than $4 \lambda$ ( $\lambda$ is the wavelength) is very long (for given phase error, its length increases with the second power of aperture length). That can be unacceptable for most applications. The horns with lenses or arrays are shorter but more complicated.

The hoghorn could act for a simple primary feed for reflector antennas (Balanis, 2008; Kuhn, 1964; Pippard, 1946; Pratt and Shearman, 1969; Schejbal, 1972). It consists of a sectoral horn flaring in only one plane and a parabolic cylinder as is shown in Fig. 1a. The paper presents numerical calculations of hoghorn dimensions. We could consider hoghorns as special variants of parabolic antennas. The hoghorns contain various advantages such as high efficiencies, excellent impedance matching, low levels of back lobe radiations, and reasonable stabilities of radiation patterns. Compact designs allow utilizations at the worst environment conditions. On the other hand, the difficulties are relatively high levels of sidelobes near by the main lobe. (Kriz, 1986; Kriz et al. 2010; Schejbal, 2011) describe various expansions of the hoghorn design such as input impedance matching for octave frequency bandwidths or dual orthogonal polarization operations. Obviously, the hoghorn can operate individually without reflector. The described methods were used for the design of certain antennas (frequency bands of 4-8, 8-12 and $12-18 \mathrm{GHz}$ ) for multilateration systems, such as Tamara and Vera passive radars, for civilian and military air traffic control (ATC), which improve the accuracy of tracking aircrafts (Bezousek and Schejbal, 2004; Schejbal et al. 1994; Slezak and Pavlovic, 2015). We compare the ample measurements with numerical results of the aperture method (Schejbal, 1972) and the full-wave numerical simulations (Vlasak and Schejbal, 2014).

\footnotetext{
${ }^{1}$ University of Pardubice, DFJP, KEEZ, Studentská 95, 53210 Pardubice, Czech Republic

2 RETIA, a.s., Pražská 341, 53002 Pardubice, Czech Republic

*corresponding author: vladimir.schejbal@upce.cz tel.: +420 466036293
} 
(Harrington, 2001; Stratton, 1941) analyze the two-dimensional radiation. The paper gives calculations of dimensions, aperture distributions and radiation patterns. That is suitable for very fast numerical computations for both analyses and syntheses. The completely new simultaneous comparisons of measurements with both aperture method and full-wave analyses allow achievement of new conclusions given in this paper.

\section{HOGHORN DIMENSIONS}

The dimension calculation uses the following equation (Schejbal, 1972)

$$
f \doteq \frac{-b_{1}+\sqrt{b_{1}^{2}-4 a_{1} c_{1}}}{2 a_{1}}
$$

where $d=B C$ is aperture height, $a=F P, F$ is parabola focus, $O F=f, \vartheta_{G}$ is the flare $A S B$ angle, $\alpha$ is the FSP angle, $\beta$ is the $F S A$ angle and $b^{\prime}=S P$ as is shown in Fig. $1 \mathrm{a}, a_{1}, b_{1}$ and $c_{1}$ are given by

$$
\begin{gathered}
a_{1}=2 \frac{\cos \vartheta_{G}+\sin \vartheta_{G}-1}{\cos \vartheta_{G}}, \\
b_{1}=a+\left(b^{\prime} \tan \vartheta_{G}-a\right) \cos \vartheta_{G}-d, \\
c_{1}=\frac{1}{4}\left[\left(a-b^{\prime} \tan \vartheta_{G}\right)^{2} \cos ^{3} \vartheta_{G}-a^{2}\right] .
\end{gathered}
$$

It is possible to determine the required parameters numerically. However, coding of the nonlinear equation solution for antenna dimensions is relatively simple.
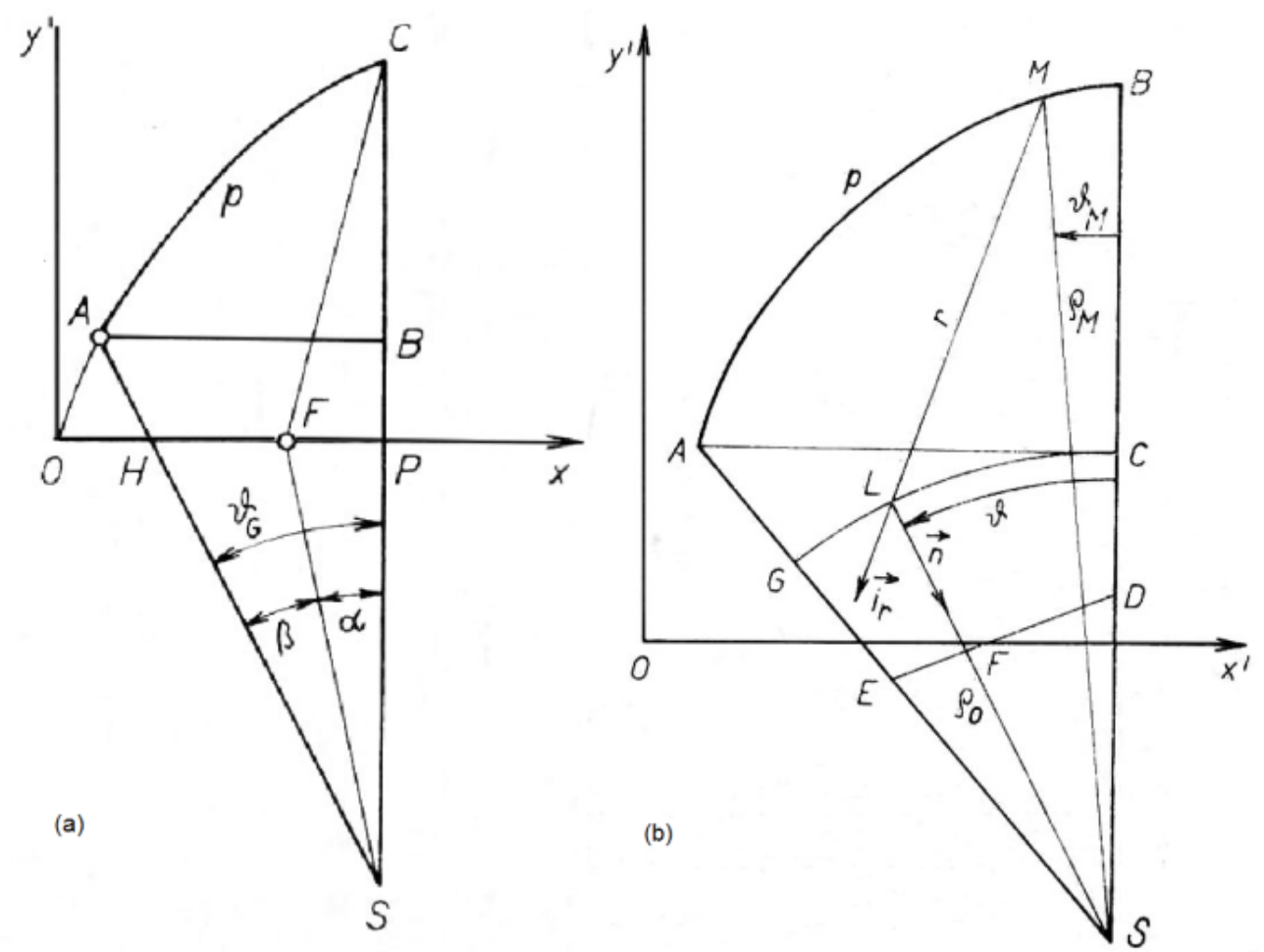

Fig. 1 Geometry (a) and field distributions (b) of hoghorn.

\section{APERTURE METHOD}

(Milligan, 2005; Silver, 1949) present the boundary value problem in wedge GSC shown in Fig. 1b. The cylindrical waves propagating between plates from arc, $G C$, to parabola, $p$, are regarded. The two- 
dimensional Kirchhoff's formula (Schejbal, 1972) calculates a parabola distribution. The fields in terms of Hankel functions in cylindrical coordinates are considered. The plane wave propagates from parabola to aperture $B C$. The resultant aperture distributions consist of a sum of radiation from arc $G C$ and the plane wave propagation from the parabola to aperture.

The following equations give the $E_{z}$ component (normal to the symmetry plane) and the $E_{y}^{\prime}$ component (parallel to the symmetry plane) of aperture distributions (Schejbal, 1972)

$$
\begin{gathered}
E_{z}=B\left\{e^{-j\left(k_{1}-\pi\right)} \int_{0}^{\vartheta_{G}}\left[H_{0}^{(2)}(k r)-j H_{1}^{(2)}(k r) \mathbf{i}_{r} \cdot \mathbf{n}\right] \sin \left(\frac{\vartheta}{\vartheta_{G}} \pi\right) d \vartheta+\right. \\
\left.+\int_{0}^{\vartheta_{G}}\left[H_{0}^{(2)}\left(k r_{2}\right)-j H_{1}^{(2)}\left(k r_{2}\right) \mathbf{i}_{r} \cdot \mathbf{n}\right] \sin \left(\frac{\vartheta}{\vartheta_{G}} \pi\right) d \vartheta\right\} \\
E_{y}^{\prime}=C\left\{e^{-j r_{1}} \int_{0}^{\vartheta_{G}}\left[H_{0}^{(2)}(\gamma r)-j H_{1}^{(2)}(\gamma r) \mathbf{i}_{r} \cdot \mathbf{n}\right] d \vartheta+\right. \\
\left.+\int_{0}^{\vartheta_{G}}\left[H_{0}^{(2)}\left(\gamma r_{2}\right)-j H_{1}^{(2)}\left(\gamma r_{2}\right) \mathbf{i}_{r} \cdot \mathbf{n}\right] d \vartheta\right\}
\end{gathered}
$$

where $k=2 \pi / \lambda, \lambda$ is the free-space wavelength and $r_{1}=x_{\mathrm{S}}^{\prime}-x_{\mathrm{M}}^{\prime}$. That corresponds to the propagation of plane wave from parabola $p$ (such as point M with $x_{M}^{\prime}, y_{M}^{\prime}$ coordinates in Fig. 1) to aperture $B C$ (with $x_{s}^{\prime}, y_{s}^{\prime}$ coordinates). Fig. 1 shows the flare angle $\vartheta_{G}$, unit vectors $\mathbf{i}_{\mathrm{r}}$ and $\mathbf{n}$. the angle $\vartheta$ corresponding to point $L$ and the distance $r$. The distance between point $L$ and the aperture point is $r_{2}, b$ is the distance between the both hoghorn plates (aperture width), $H_{0}{ }^{(2)}(k r)$ and $H_{1}{ }^{(2)}(k r)$ are Hankel functions of the second kind of order 0 and 1, respectively, and $\gamma=\left[k^{2}-(\pi / b)^{2}\right]^{1 / 2}$. The integrals in equations (2) and (3) are calculated using $n$ point Gaussian integration, whose idea is to give the freedom to choose weighting coefficients but also locations of points. The integration is exact for all polynomials of degree $2 n-1$ or less. The radiation patterns are determined using computed aperture distributions (2) or (3) by an aperture method (Schejbal, 1972).

\section{COMPARISONS OF NUMERICAL SIMULATIONS AND MEASUREMENTS}

The aperture methods and full-wave numerical simulations have been used for the hoghorn design with $\vartheta_{G}=50^{\circ}$ and $256 \times 30 \mathrm{~mm}$ hoghorn mouth for both polarizations. That means for components $E_{z}$ (normal) and $E_{y}^{\prime}$ (parallel). We compare the computations using the aperture method and the full-wave simulations with the measurements of the far field patterns and field distributions. For a relatively narrow frequency band of $9.25-9.45 \mathrm{GHz}$, the design could be rather simple. We can roughly estimate an admittance of sectoral horn from the transmission-line point of view (Silver, 1949). The sectoral horn consists of a length of sectoral guide terminated by the mouth admittance at one end and joined to uniform guide at the other. We discus the hoghorn admittance characteristics considering sectoral guide transmission-line arguments. The full-wave numerical simulations usually allow much better analyses of admittances.

The magnitude of scattering parameter $S_{11}$ (reflection coefficient) show Fig. 3 in (Vlasak and Schejbal, 2014) for both polarizations (normal and parallel to the symmetry plane), and therefore is not shown here. It is clear that the reflection coefficients for both polarizations are relatively low. Moreover, the frequency bandwidths are quite broad and it is possible to extend them easily. The correspondence to measurements are not performed since the measurement has been done with a transition between standard rectangular waveguide and square cross section of hoghorn input and numerical simulations have been done without any transition. That would create some discrepancies considering very low reflection coefficients of the transition and hoghorn. Similarly, the comparison with rather rough estimation using the transmissionline point of view is not very useful. 
Fig. 2a shows the hoghorn and 3D radiation pattern of normal polarization, $E_{z}$, for frequency of $9.375 \mathrm{GHz}$ using full-wave numerical simulations. Similarly, Fig. $2 \mathrm{~b}$ shows the hoghorn and 3D radiation pattern of parallel polarization, $E_{y}^{\prime}$, for the same frequency. Considering Figs. $2 \mathrm{a}$ and $2 \mathrm{~b}$ the directivities can be seen. The normal polarization directivity for $E_{z}$ is $18.9 \mathrm{dBi}$ and the parallel polarization directivity for $E_{y}^{\prime}$ is 17.4 $\mathrm{dBi}$. The patterns offer the directivity. Gain and directivity vary only by the efficiency. Therefore, it is necessary (Milligan, 2005) to measure the gain, i.e. product of directivity and efficiency.
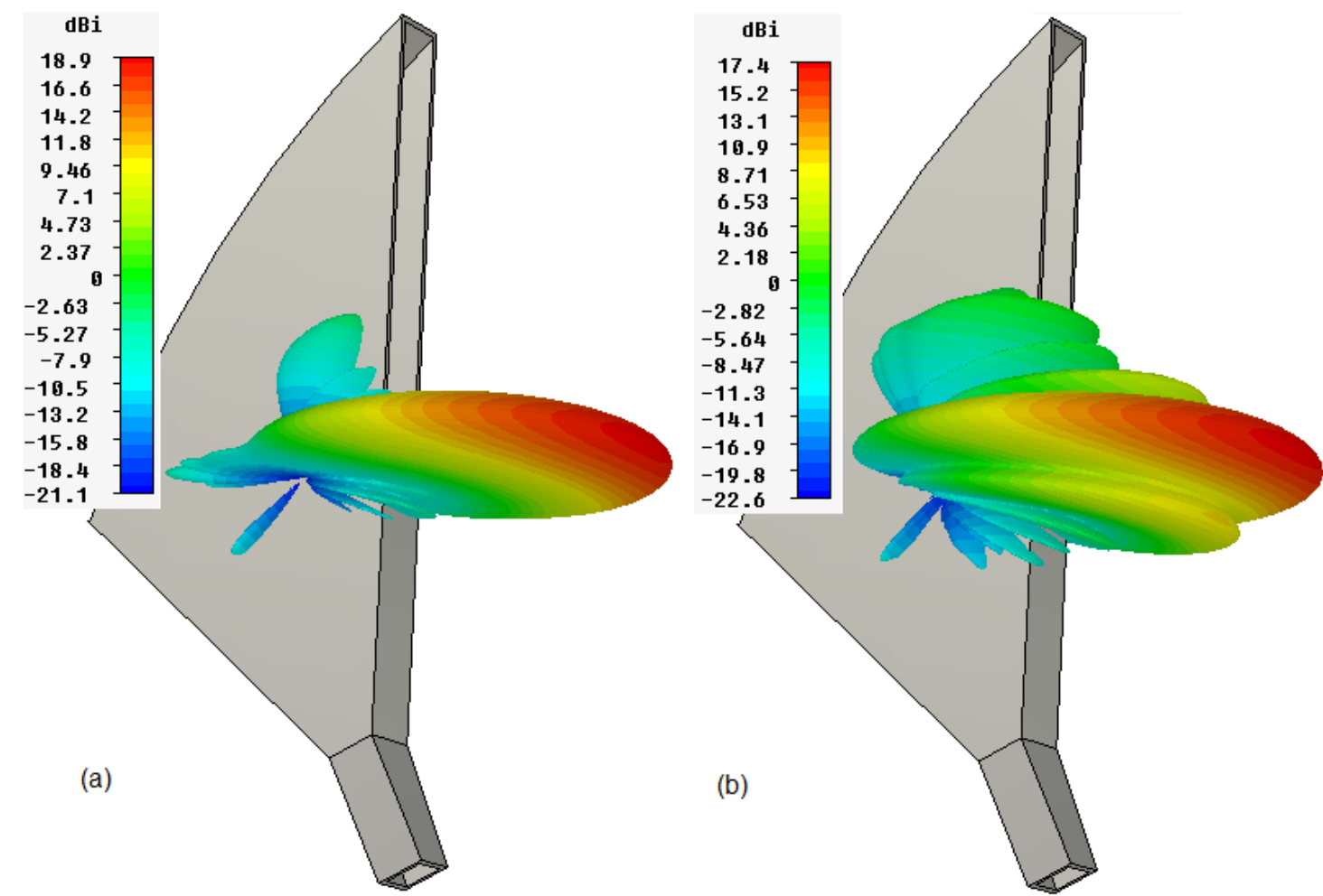

Fig. 2 The 3D radiation pattern for normal polarization, $E_{z}$ (a) and parallel polarization, $E_{y}^{\prime}$, (b).

We have measured the radiation patterns at an anechoic chamber with a $4 \mathrm{~m}$ separation between both antennas. It is clear that the measurements are not very accurate, as this separation creates quadratic phase errors (the usual criterion $2 d^{2} / \lambda$ gives about $4.1 \mathrm{~m}$ ). Normally, we should use much greater distance for accurate measurements. We have employed the comparison technique using standard horn antenna (Hollis et al. 1985) for "far-field" gain measurement. Calculated gains suffice for some comparison measurements where high accuracy is not required. We have to take an attention to obtain a proper test environment and processing the errors from various sources to insure that the measured gain reveals the true gain of antenna. The measured gains are approximately $18 \mathrm{dBi}$ and slightly higher gain by $0.3 \mathrm{~dB}$ for the normal polarization.

The error of gain measurements could be estimated as $0.5 \mathrm{~dB}$ for a given far-field range. We have to consider not only the antenna under test but the standard horn antenna. On the other hand, the electric field distribution, and therefore radiation pattern directivities found by numerical simulations, are disturbed in the plane normal to the symmetry plane (Dolecek and Schejbal, 2009; Schejbal, 1999). Frequently, the numerical simulations of radiation patterns for narrow aperture are not very accurate. Therefore, it could be concluded that the directivities found by numerical simulations agree with the measurements of gain as the efficiency of hoghorn is very high. 
Fig. 3 shows the calculation (2), numerical simulations, and measurement of radiation pattern for normal polarization of $9.375 \mathrm{GHz}$ frequency. Similarly, Fig. 4 shows the calculation (3), numerical simulations and measurement of radiation pattern for parallel polarization for the same frequency. The experiments are shown by solid lines, calculations (2) or (3) by crosses, and numerical simulations are shown by dotted lines in Figs. 3 and 4. This demonstrate that the measured values correspond to numerical simulations and calculations. The agreement is better for normal polarization, $E_{z}$.

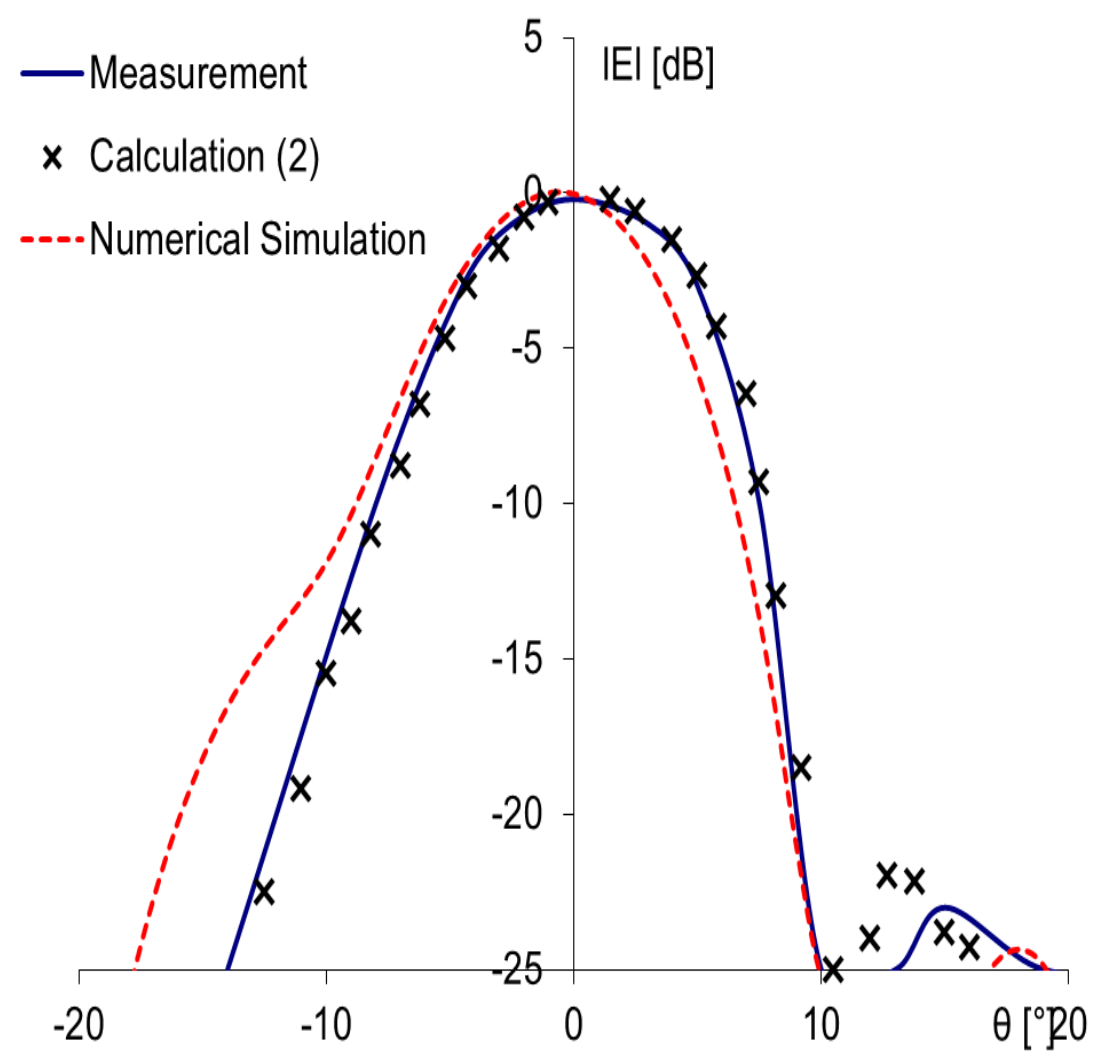

Fig. 3 Calculation (2), numerical simulations, and measurement of radiation pattern for normal polarization, $E_{z}$,

$(f=9.375 \mathrm{GHz})$.

It is possible to explain partly the greater differences of parallel polarization considering the tolerances. The hoghorn contain a parallel plate waveguide with the distance $b$ between plates (Harrington, 2001). If we analyze the change of $b$ then the phase change for a distance $l$ is

$$
\frac{d \varphi}{d b}=\frac{d\left[\frac{2 \pi}{\lambda_{g}} l\right]}{d b}=\frac{d\left[\frac{2 \pi}{\lambda} l \sqrt{1-\left(\frac{\lambda}{2 b}\right)^{2}}\right]}{d b}=-\frac{\pi l \lambda_{g}}{2 b^{3}}
$$

where $\lambda$ is the free-space wavelength and $\lambda \mathrm{g}$ is the guide wavelength. Considering $\mathrm{l}=380 \mathrm{~mm}, \mathrm{~b}=30 \mathrm{~mm}$ and width change of $\Delta \mathrm{b}=0.5 \mathrm{~mm}$ the phase change would be 24 degrees. That could cause the radiation pattern changes such as shifting of beam directions or shape deformations due to phase shift between the direct radiation from the input horn and reflection from the parabolic cylinder. It is obvious that these changes would create frequency instabilities.

Similarly, we can perform the stability analyses using (2) and (3). We have tested the field distributions using the center-fed half-wavelength dipole with the $2 \mathrm{~mm}$ distance in front of the aperture. (Schejbal, 2011) perform the detailed analyses. Thus, the substantial outcomes are only given.

We can see that the measured distributions of $E_{z}$ normal components for different frequencies do not change substantially. Measured and calculated results are very similar. 
However, we can deduce that the measured distributions of $E_{y}^{\prime}$ parallel components for different frequencies change substantially. Measured and calculated results are substantially different. The interferences of incident radiation from arc $G C$ and the plane wave propagation from parabola to aperture (3) mostly cause discrepancies as they shift the maxima and minima positions. Moreover, the probes operate as spatial filters on the near-field phase front.

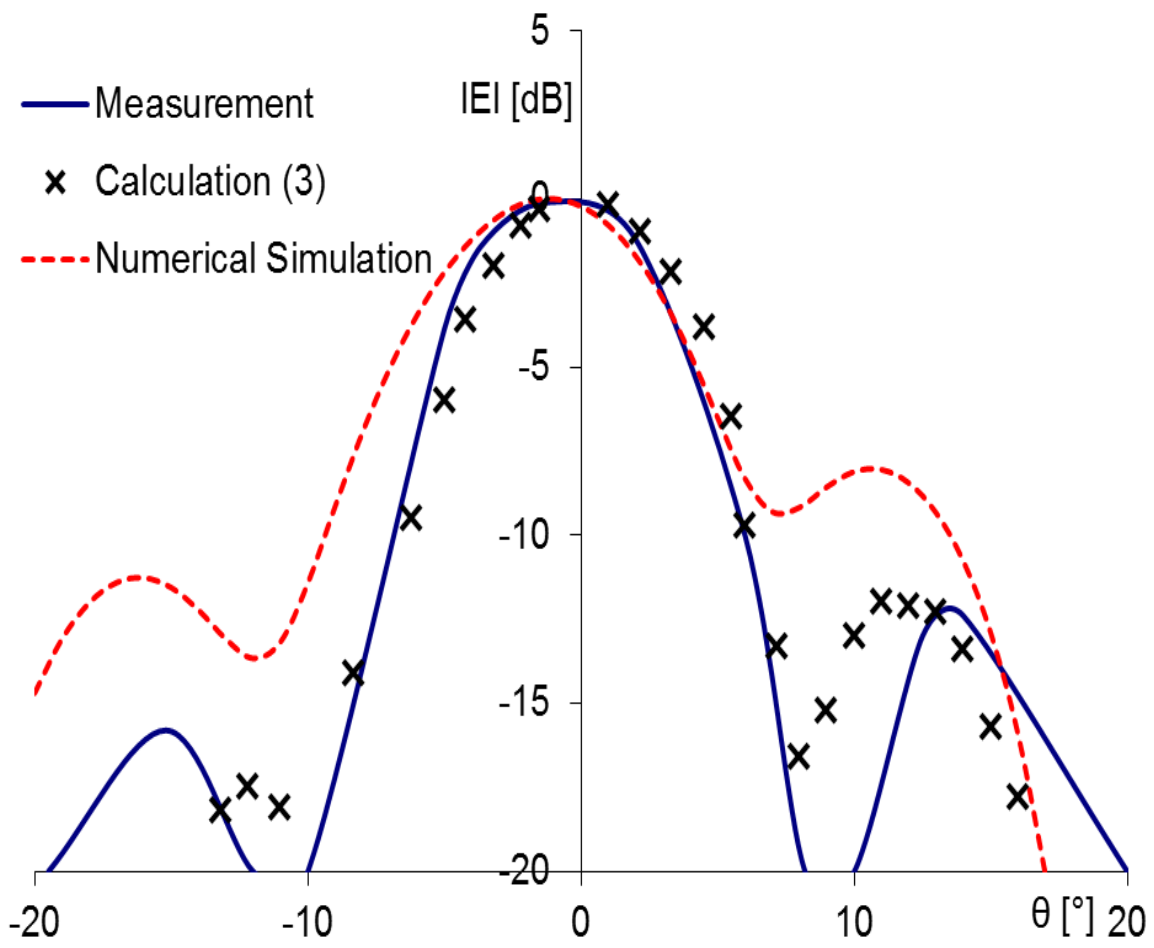

Fig. 4 Calculations (3), numerical simulations and measurement of radiation pattern for parallel polarization, $E_{y}^{\prime},(f=9.375 \mathrm{GHz})$.

To diminish (and prove experimentally) reflections at point $A$ shown in Fig. 1a for parallel polarizations, we place two plates parallel to the symmetry according to Fig. 6 in (Schejbal, 2011). The theoretical analyses of effects of inserted plates could be very complicated but it is possible to assume that effects of inserted plates for normal polarizations are very small. On the other hand, the parallel polarizations cannot propagate between inserted plates, when operating frequency is less than cutoff frequency. That means none of modes can propagate, and therefore the reflection at point $A$ cannot occur, i.e. an effective wall reflects rays at various directions. The matched dual-mode square waveguide corner use the same idea of effective wall (Park and Eisenhart, 1989; Schejbal, 1972).

The radiation patterns of hoghorn with inserted plates with normal polarizations are not changed but for parallel polarization, $E_{y}^{\prime}$, are much more stable (as Fig. 7 and 8 in (Schejbal, 2011) demonstrate). We can clarify that using the comparison of beamwidths for the original hoghorn and the hoghorn with inserted plates. Fig. 5 shows the measured values of beamwidths for $-10 \mathrm{~dB}$ levels. The IP labels indicate the inserted plate beamwidths.

It is not necessary to state that the accuracy of radiation pattern calculations using aperture method (2) or (3) for hoghorn with inserted plates would be very low. Therefore, we do not show these radiation patterns. On the other hand full-wave numerical simulations may be successfully used. Fig. 6 shows the 3D radiation pattern for normal polarization, $E_{z}$ and parallel polarization $E_{y}^{\prime}$.

According to measurements and full-wave numerical simulations, we can clearly demonstrate that the radiation patterns for normal polarizations are relatively stable for both cases (i.e. with and without inserted plates). However, the radiation patterns for parallel polarizations are slightly changing. That changes the beamwidths, sidelobe levels, and therefore the gain differences (Dolecek and Schejbal, 2009). 
On the other hand, we can observe that the inserted plates substantially improved the radiation frequency stability. However, the inserted plates cannot improve the changes of radiation patterns such as shifting of beam directions or shape deformations due to phase shift between the direct radiation from the input horn and reflection from the parabolic cylinder for parallel polarization, $E_{y}^{\prime}$, caused by the tolerances (4).

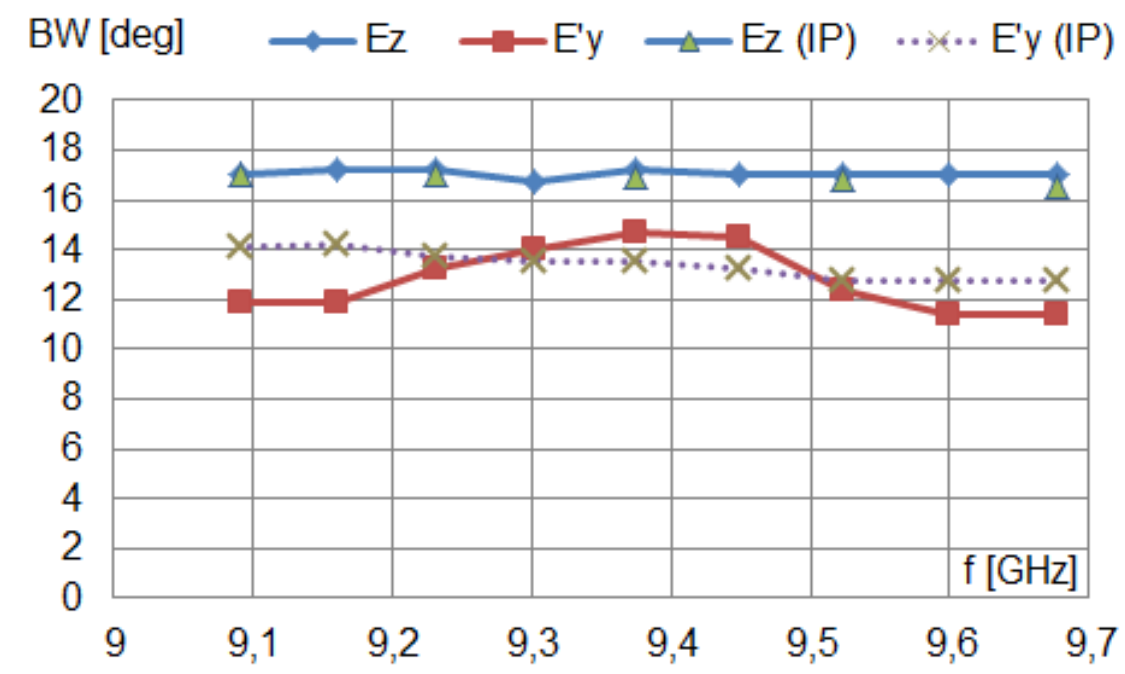

Fig. 5 Measured values of beamwidths for $-10 \mathrm{~dB}$ levels for polarizations normal $\left(E_{z}\right)$ and parallel $\left(E_{y}^{\prime}\right)$ to the symmetry plane. Inserted plates are labelled by IP.
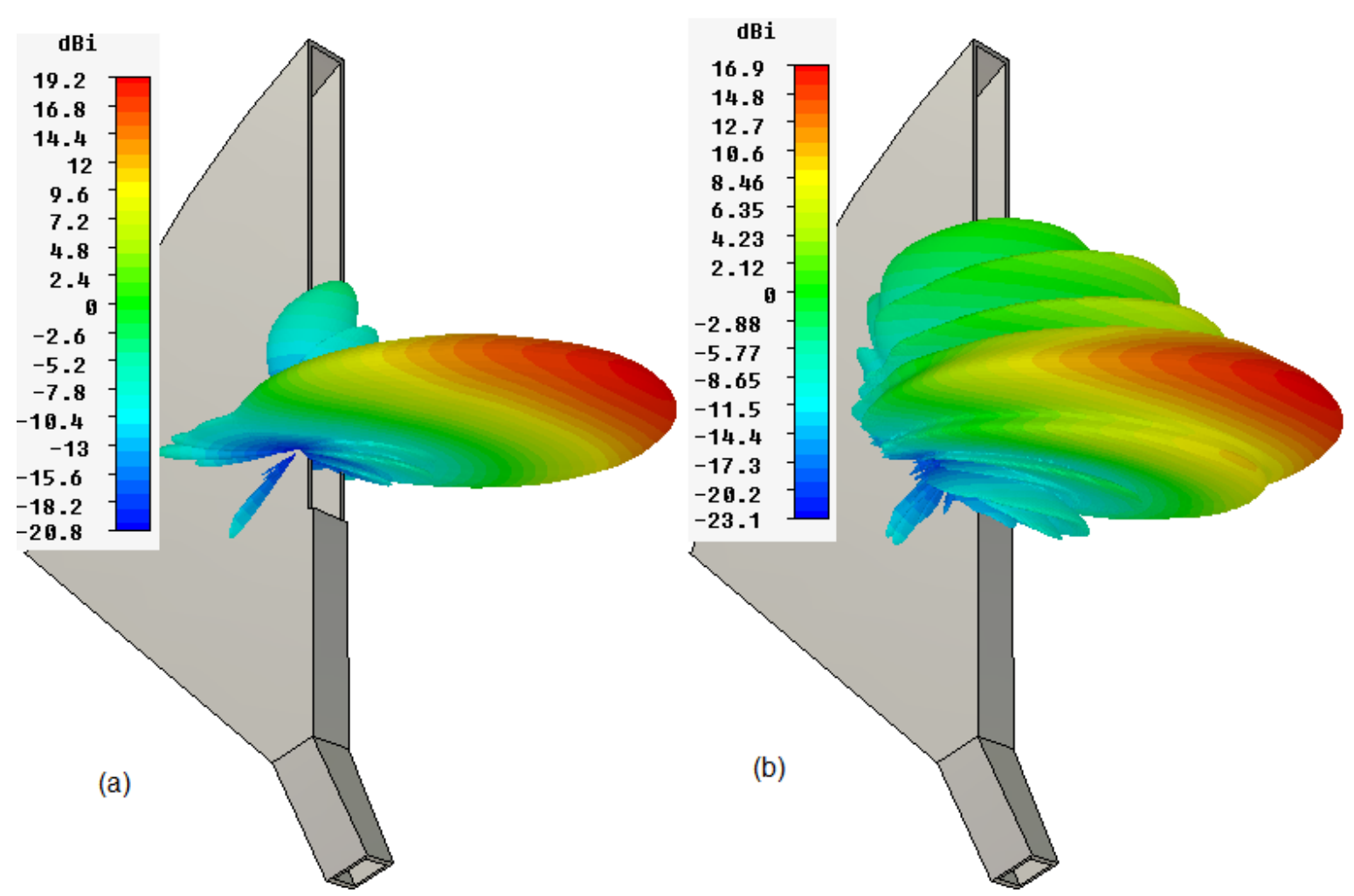

Fig. 6 The 3D radiation pattern of hoghorn with inserted plates for normal polarization $E_{z,}(\mathrm{a})$ and parallel polarization $E_{y}^{\prime}(\mathrm{b})$.

It is obvious that these changes would create frequency instabilities. That cannot discover aperture method or numerical simulation without any tolerance analysis. The aperture method, which is very fast, could perform this more advantageously (much more quickly). Similarly, the aperture method could be advantageously used for syntheses and/or optimizing of hoghorns. An example of optimization considering the change of $\alpha+\beta$ angles (see Fig. 1) gives Tab. 1. It is not necessary to explain that detailed 
observations such as the field distribution analyses and/or radiation patterns could reveal various physical phenomena, which could affect the hoghorn design. Obviously, detailed observation of electric and/or magnetic field distributions using the full wave numerical simulations could be very useful and the combination of both aperture method and numerical simulations would improve the physical understanding of the antenna design.

Tab. 1 Optimization of $\alpha+\beta$ angles for normal polarizations.

\begin{tabular}{cccccc}
\hline $\boldsymbol{\alpha}+\boldsymbol{\beta}$ (deg) & $\mathbf{4 0}$ & $\mathbf{6 0}$ & $\mathbf{7 0}$ & $\mathbf{7 5}$ & $\mathbf{8 0}$ \\
\hline $3 \mathrm{~dB}$ beamwidth $(\mathrm{deg})$ & 11.5 & 13.7 & 11.5 & 12.7 & 13.3 \\
$10 \mathrm{~dB}$ beamwidth $(\mathrm{deg})$ & 19.6 & 27.4 & 19.8 & 21.7 & 23.4 \\
Sidelobe $(\mathrm{dB})$ & 15.6 & 15 & 16.2 & 17 & 15 \\
\hline
\end{tabular}

We can consider the uniform distribution for normal components, $E_{z}$, and the cosine distribution of the parallel component, $E_{y}^{\prime}$, for radiation pattern in plane normal to the symmetry plane. However, the distance between the hoghorn plates is about a wavelength, and therefore the aperture method calculation is not suitable as aperture theory fails for small horns as the edge diffractions determine beams much more than the aperture fields (Kriz at el. 2010; Silver, 1949).

\section{CONCLUSION}

We have used the described methods for the design of certain antennas (frequency bands of 4-8, $8-12$ and $12-18 \mathrm{GHz}$ ) for multilateration systems, such as Tamara and Vera passive radars, for civilian and military air traffic control, which greatly improve the accuracy of tracking aircrafts.

We perform the analyses of hoghorn using two various approaches: an aperture method and full-wave numerical simulations. The paper explain calculations of hoghorn dimensions, aperture distributions and radiation patterns using the aperture method. That is very suitable for numerical calculations considering the CPU time and memory. The two-dimensional Kirchhoff's formula can analyze parabola distribution. The field calculations in cylindrical coordinates use the Hankel functions.

We calculate the radiation patterns by an aperture method using aperture distributions. It is obvious that this procedure is very simplified as aperture method could compute the structures substantially greater than the wavelength. Moreover, we use various simplifications such as neglecting of reflections from abrupt changes inside hoghorn.

We compare the numerical results of the aperture method and the full-wave numerical simulations with the experiments. This enables to obtain new conclusions. Fig. 5 shows slight discrepancies for one polarization, which the proposed improvements diminish.

We could conclude that the directivities found by numerical simulations agree within the estimated errors with gain measurements of the hoghorn. Actually, the differences between gain and directivity are very small as the efficiency of hoghorn is very high.

The measurements of radiation patterns are consistent with numerical simulations. The agreement is better for polarization normal to the symmetry plane than for polarization parallel to the symmetry plane.

It is possible to explain the greater differences of polarization parallel to the symmetry plane considering the tolerances. Moreover, the reflections are especially important considering point $A$, shown in Fig. 1 (the horn and parabola joint). To diminish (and prove experimentally) these reflections for $E_{y}^{\prime}$ parallel components, we propose the changes of the hoghorn design. That diminishes reflections at this junction for the parallel polarization. 
We have demonstrated that both the aperture method and full-wave numerical simulations enable the hoghorn design. The new simultaneous comparisons of measurements with both aperture method and full-wave analyses allow reaching new conclusions given in this paper. The aperture method is very fast. Therefore, it could be advantageously used for syntheses and/or optimizing of hoghorns. On the other hand, the full-wave numerical simulations are user friendly and can offer more possibilities (some of them are clearly illustrated) but they are usually time-consuming.

It is not necessary to state that the accuracy of radiation pattern calculations using aperture method for improved hoghorn with inserted plates could be very low, and therefore it is not possible to use them successfully.

Alternatively, we could use the full-wave numerical simulations even for very complicated situations as Fig. 6 demonstrates. Obviously, detailed observation of electric and/or magnetic field distributions using the full wave numerical simulations could be very useful and the combination of both aperture method and numerical simulations would improve the physical understanding of the antenna design.

\section{References}

Balanis, C. A. 2008. Modern Antenna Handbook. Hoboken: John Wiley \& Sons.

Bezousek, P. and Schejbal, V. 2004. Radar technology in the Czech Republic. IEEE Aerospace and Electronic Systems Magazine, 19 (8), pp. 27-34.

Dolecek, R. and Schejbal, V. 2009. Estimation of Antenna Gain. IEEE Antennas and Propagation Magazine, 51 (1), pp. 124-125.

Harrington, R. F. 2001. Time-Harmonic Electromagnetic Fields. New York: John Wiley \& Sons.

Hollis, J. S., Lyon, T. J. and Clayton, L. 1985. Microwave Antenna Measurements. Atlanta: Scientific-Atlanta.

Kriz, J. 1986. Sectoral horn-reflector antenna with low side lobes. Slaboproudy obzor, 47 (1], pp. 9 - 13.

Kriz, J., Krcmar, V., Pidanic, J. and Schejbal, V. 2010. Antenna beamwidth control. IEEE Antennas and Propagation Magazine, 52 (1), pp. 163-170.

Kuhn, R. 1964. Mikrowellenantennen. Berlin: VEB Verlag Technik.

Milligan, T. A. 2005. Modern Antenna Design. Hoboken: John Wiley \& Sons.

Park, P. K. and Eisenhart, R. L. 1989. Matched dual mode waveguide corner. US patent no. 4795993 A.

Pippard, A. B. 1946. The Hoghorn - an electromagnetic horn radiator of medium-sized aperture. Proc. IEE, 93, Pt III. A (10), pp. 1536-1538.

Pratt, T. and Shearman, E. D. R. 1969. Sectoral Hoghorn: A new Form of Line Feed for Spherical Reflector Aerials. Electronics Letters, 5 (1), pp. 1-2.

Schejbal, V. 1972. Hoghorn. Slaboproudy obzor 33 (2), pp. 57 - 65.

Schejbal, V. 1972. Waveguide Corner. Czechoslovakia patent no. AO 149814.

Schejbal, V. et al. 1994. Czech radar technology. IEEE Trans. on Aerospace and Electronics Systems, 30 (1), pp. 2-17.

Schejbal, V. 1999. Directivity of planar antennas. IEEE Antennas and Propagation Magazine. 41 (2), pp. 60 -62 .

Schejbal, V. 2011. Hoghorn improved design. The 21st International Conference Radioelektronika 2011. Brno: IEEE, pp. 163-166.

Silver, S. 1949. Microwave Antenna Theory and Design. New York: McGraw-Hill. 
Slezak, L. and Pavlovic. M. 2015. TDOA based PET antennas: History and present status. Conference on Microwave Techniques (COMITE) 2015. Pardubice, Czech Rep. 2015, pp. 1-4.

Stratton, J. A. 1941. Electromagnetic Theory. New York: McGraw-Hill.

Vlasak, V. and Schejbal, V. 2014. Numerical simulation for hoghorn design. 24th International Conference Radioelektronika 2014. Bratislava: IEEE, pp. 1-4. 